\title{
Solvent uptake and cavitation
}

\author{
Fernando P. Duda ${ }^{a}$, Angela C. Souza ${ }^{b}$, Eliot Fried ${ }^{\mathrm{c}, *}$ \\ a Programa de Engenharia Mecânica, COPPE/UFRJ, Rio de Janeiro, Brazil \\ b Departamento de Engenharia Mecânica, PGMEC/UFF, Niterói, Brazil

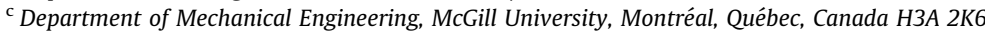

\section{A R T I C L E I N F O}

\section{Article history:}

Received 21 February 2011

Received in revised form

27 July 2011

Accepted 8 August 2011

Available online 16 August 2011

\section{Keywords:}

Gel

Multiphase equilibria

Swelling

Energy minimization

Stability

\begin{abstract}
A B S T R A C T
This paper offers a treatment for investigating the role of the interplay between mechanics and the absorption of a swelling agent on the equilibrium and cavitation of a spherical specimen. This treatment is then applied, within the context of the classical theory of swelling of a rubber by a liquid, to establish, among other things, necessary conditions for the existence of multiphase equilibria and the occurrence of cavitation. These conditions involve the parameters entering the theory, namely the number $c_{S}$ of polymer chains per unit reference volume, the volume $v$ occupied by a swelling-agent molecule, and the Flory-Huggins interaction parameter $\chi$.
\end{abstract}

(c) 2011 Elsevier Ltd. All rights reserved.

\section{Introduction}

The interaction of mechanics and absorption of a swelling agent arises in a variety of contexts involving solids. In particular, it underlies many important phenomena in polymeric solids and gels. Among these, we mention environmental stress cracking (Scheirs, 2000), anomalous diffusion (Crank, 1990) and phase transformations (Onuki, 1993). Many continuum theories concerning this kind of interaction have been developed over the years, giving rise to an abundant literature on the subject (see, for instance, the recent contributions of Barriere and Leibler, 2003; Rajagopal, 2003; Hong et al., 2008; Doi, 2009; Duda et al., 2010; Chester and Anand, 2010; Baek and Pence, 2011; and references cited therein).

Building upon the framework of Duda et al. (2010), we explore the interaction of mechanics and absorption of a swelling agent on the existence of multiphase equilibria and the occurrence of cavitation in elastic spheres. More specifically, we consider an initially dry and intact, that is, not hollow, elastic sphere undergoing a spherically symmetric deformation and swelling induced by the combined action of mechanical loads, defined in terms of the ambient pressure and a normal Piola traction, and the absorption of a swelling agent. The swollen sphere is viewed as a two-component body composed of two incompressible constituents, namely the elastic solid and the swelling agent. The state of the swollen sphere is, therefore, fully characterized by the distribution of the swelling agent and the radius of a central hole. If the radius is zero, there is no hole and the swollen sphere remains intact. When the ambient chemical potential of the swelling agent is fixed and chemical equilibrium prevails at the boundary between the solid and the environment, we describe two types of intact states. In the first of these, the sphere inhabits a uniform equilibrium state. In the second of these, the swelling-agent concentration is piecewise uniform within the sphere. This includes the case of a sphere divided

\footnotetext{
* Corresponding author.

E-mail addresses: duda@mecanica.coppe.ufrj.br (F.P. Duda), angelacristinacs@uol.com.br (A.C. Souza), eliot.fried@mcgill.ca (E. Fried).
} 
in two regions, the inner one dry and the outer one uniformly swollen, separated by a sharp boundary. For these states, we also investigate the possibility of cavitation - that is, the sudden appearance of a central traction-free cavity. Of importance in the analysis presented here is the notion of net potential energy, the definition of which requires the specification of the chemical potential of the environment and the provision of a constitutive relation for the free-energy density of a solventinfused solid. It is important to clarify that the cavitation scenario envisaged here is based on the assumption that the transformation from an intact state to a cavitated state occurs very rapidly. In harmony with this assumption, the solvent content remains the same before and after the occurrence of cavitation. Therefore, the solvent concentration entering the cavitation analysis is identical to that of the intact state, which depends on the applied Piola traction $\sigma$.

A detailed analysis is presented for the special case in which the environment is a pure and incompressible fluid bath and the free-energy density is chosen in consonance with the classical theory of swelling of rubbers (Treloar, 1975). In particular, we investigate the effects of the constitutive parameters entering the theory-namely the number $c_{S}$ of polymer chains per unit reference volume, the volume $v$ occupied by a swelling-agent molecule, and the Flory-Huggins interaction parameter $\chi$-on the characteristics of the two types of intact states. For uniform equilibrium states, multiphase equilibria and cavitation are impossible unless the parameter $\chi$ exceeds a certain critical value depending on $c_{S} v$ and, granted that cavitation occurs, the ratio $v p_{a} / k_{B} T$, where $p_{a}$ is the environmental pressure of the solvent, $k_{B}$ is Boltzmann's constant, and $T$ is the absolute temperature. These critical values both increase with $c_{S} v$, a parameter which describes the relative importance of the elastic and mixing contributions to the free-energy density, and are greater than $1 / 2$. Therefore, neither multiphase equilibria nor cavitation is possible if the sphere is immersed in a good solvent, since in this case $\chi \leq 1 / 2$. As for states with a dry core surrounded by a swollen annular zone, we show that cavitation may occur even for non-positive values of the applied Piola traction $\sigma$. Whether this is possible depends on the values of the interaction parameter $\chi$ and the interface location $R_{*}$.

It is worth mentioning that the occurrence of multiphase equilibria indicates the possibility of modeling polymeric gels that are capable of sustaining volumetric-phase transformations. Subsequent to its theoretical prediction by Dusek and Patterson (1968) and experimental observation by Tanaka (1978), this type of phase transformation has been the subject of many theoretical and experimental works. Consequently, there is a vast literature on the subject and the interested reader is referred to the survey articles by Onuki (1993), Shibayama and Tanaka (1993), Li and Tanaka (1992), Hirotsu (1994), and Doi (2009). However, it is worth noting that the special case presented here, which is based on the Treloar (1975) theory of swelling, precludes phase transformations during free swelling. The same situation prevails within the context of the Flory (1953) theory of swelling, which differs from the Treloar (1975) theory by a logarithmic volumetric contribution to the elastic energy, unless, as Erman and Flory (1986) show, the interaction parameter $\chi$ is dependent on the solvent concentration. Alternatively, Hirotsu (1994) shows that, due to the presence of counterions, the Flory (1953) theory for the swelling of ionized gels predicts that volumetric phase transformations are possible even if $\chi$ is concentration independent, as is the case considered here. In this regard, an applied Piola traction can be viewed as generating an effective supply of counterions.

It is worth emphasizing that cavitation is an important failure mode in elastomeric materials (Fond, 2001), which includes situations where failure is mediated by environmental factors (e.g., Gent (1970), Saylor et al. (2010), and Werner (2010)). Of particular importance is the work of Gent and Lindley (1959), who conducted experimental investigations showing that the critical load for cavitation was directly related to the elastic modulus. Gent and Lindley (1959) viewed cavitation as the result of unlimited elastic expansion of a pre-existing microcavity and used the elastic theory of cavity inflation to successfully correlate the critical load for cavitation with the corresponding one necessary for the unbounded growth of a cavity in an infinite medium. An alternative interpretation was advanced by Ball (1982), who viewed cavitation as the generation of a traction-free hole in the interior of an apparently solid body subsequent to the attainment of a critical load. In both views, cavitation is considered to be a problem in nonlinear elasticity. For a review of cavitation in that context see, for example, the article of Horgan and Polignore (1995). The approach adopted here is similar to the treatment of cavitation of swollen spheres presented by Pence and Tsai (2006), who advanced the work of Ball (1982) to account for the presence of a given degree of swelling. Our approach builds upon that of Pence and Tsai (2006) by accounting for load-dependent swelling caused by solvent uptake.

The remainder of this paper is organized as follows. Basic concepts are introduced in Section 2. A general framework for studying the uptake of a solvent by an elastic sphere is presented in Section 3. Cavitation is discussed in Section 4. Simple applications are considered in Section 5. Finally, concluding remarks are provided in Section 6.

\section{Preliminaries}

Consider the absorption of a swelling agent, or, for brevity, solvent, by a solid body $\mathcal{B}$ identified with its placement in a fixed reference configuration. As a result, the solid undergoes two interdependent processes: a mechanical (macroscopic) processes due to deformation and a chemical (microscopic) process due to solvent absorption, transport, and accumulation. The state of $\mathcal{B}$ at time $t$ is then defined in terms of its deformation $\mathbf{y}(\cdot, t)$ and solvent concentration $c(\cdot, t)$, which respectively assign to each material point $\mathbf{X}$ in $\mathcal{B}$ the corresponding spatial location and solvent content per unit reference volume at time $t$.

We now present equations that govern the uptake of a solvent by $\mathcal{B}$ under the action of mechanical loads. This set of equations, which is a particular version of the general equations of Duda et al. (2010), is comprised by the constraint of 
incompressibility, which represents the classical assumption that the solid and solvent are both incompressible, and two field equations that describe the mechanical and chemical features of the problem. The equations are conveniently organized as follows:

- Incompressibility constraint

$$
\operatorname{det} \mathbf{F}=1+v c \text {. }
$$

- Mechanics

$$
\operatorname{Div} \mathbf{S}=\mathbf{0}, \quad \mathbf{S}=\frac{\partial \hat{\psi}(\mathbf{F}, c)}{\partial \mathbf{F}}-q \mathbf{F}^{-\top}
$$

- Chemistry

$$
\frac{\partial c}{\partial t}=-\operatorname{Div} \mathbf{J}, \quad \mathbf{J}=-\hat{\mathbf{M}}(\mathbf{F}, c) \nabla \mu, \quad \mu=\frac{\partial \hat{\psi}(\mathbf{F}, c)}{\partial c}+\frac{v q}{1+v c} .
$$

Here and henceforth, $\mathbf{F}=\nabla \mathbf{y}$ is the deformation gradient, $v$ is the volume occupied by a solvent molecule, $\mathbf{S}$ is the Piola stress tensor, $\hat{\psi}$ and $\hat{\mathbf{M}}$ are the constitutive response functions determining the free-energy density and the solvent mobility, $q$ is a Lagrange multiplier field associated with the constraint (1), $\mathbf{J}$ is the solvent flux relative to the solid, and $\mu$ is the chemical potential of the solvent in the solid. Body force, inertial effects, and solvent supply are neglected here. Importantly, mechanics and chemistry are coupled via the incompressibility constraint. Additional couplings may also arise through the response functions $\hat{\psi}$ and $\hat{\mathbf{M}}$.

Along with the aforementioned governing equations, initial and boundary conditions must also be imposed. Whereas the former involves the prescription of the initial distribution of the solvent concentration $c$, the latter requires the prescription of conditions that describe interactions between the solid and the environment. Here, we suppose that $\mathcal{B}$ is immersed in an environment wherein the chemical potential of the solvent is constant and equal to $\mu_{a}$ and assume that at the interface $\partial \mathcal{B}$ between the solid and the environment a traction $\mathbf{s}$ is prescribed and chemical equilibrium prevails, that is, that

$$
\mathbf{S n}=\mathbf{s}, \quad \mu=\mu_{a},
$$

where $\mathbf{n}$ is the unit normal to $\partial \mathcal{B}$.

Observe that (2) expresses mechanical equilibrium. Chemical equilibrium, on the other hand, requires that $c$ be timeindependent and that $\mathbf{J}=\mathbf{0}$. These imply that (3) is trivially satisfied and that $(3)_{2}$ reduces to $\nabla \mu=\mathbf{0}$. For the environment under consideration, this condition and $(4)_{2}$ imply that

$$
\mu=\mu_{a}
$$

on $\mathcal{B}$. Mechanochemical equilibrium states are therefore governed by (1), (2), (3) 3 , and (5), along with the boundary condition $(4)_{1}$. That (5) holds on $\mathcal{B}$ ensures satisfaction of $(4)_{2}$. Further, if the mechanical loading is conservative, that is, if there exists an external potential energy $\mathcal{L}[\mathbf{y}]$ for the mechanical load, mechanochemical equilibrium states correspond to critical points of the net potential energy functional of $\mathcal{B}$

$$
\mathcal{F}[\mathbf{y}, c]=\int_{\mathcal{B}}\left(\hat{\psi}(\mathbf{F}, c)-\mu_{a} c\right) d V+\mathcal{L}[\mathbf{y}]
$$

subject to the constraint (1). On the basis of the energy criterion for stability, an equilibrium state is stable if it is a local minimum of $\mathcal{F}$ and otherwise is unstable.

We assume that $\hat{\psi}$ has the form

$$
\hat{\psi}(\mathbf{F}, c)=\tilde{\psi}\left(I_{1}(\mathbf{B}), I_{2}(\mathbf{B}), c\right),
$$

where $\mathbf{B}:=\mathbf{F F}^{\top}$ is the left Cauchy-Green tensor and $I_{1}(\mathbf{B})=\operatorname{tr} \mathbf{B}$ and $I_{2}(\mathbf{B})=\left(I_{1}^{2}(\mathbf{B})-I_{1}\left(\mathbf{B}^{2}\right)\right) / 2$ are its first and second principal invariants. It can be shown that this representation holds for the case of isotropic behavior. In this case, the relation (2) 2 determining the Piola stress $\mathbf{S}$ specializes to

$$
\mathbf{S}=\alpha_{1} \mathbf{F}+\alpha_{2}\left(I_{1}(\mathbf{B}) \mathbf{I}-\mathbf{B}\right) \mathbf{F}-q \mathbf{F}^{-\top},
$$

where $\alpha_{i}=2 \tilde{\psi}_{i}\left(I_{1}(\mathbf{B}), I_{2}(\mathbf{B}), c\right)$ and $\tilde{\psi}_{i}\left(I_{1}, I_{2}, c\right)=\partial \tilde{\psi}\left(I_{1}, I_{2}, c\right) / \partial I_{i}$.

It is expected that obtaining states of $\mathcal{B}$ by solving directly the aforementioned equations is not an easy task, even for simple geometries, loading conditions, and choices of the constitutive response functions. Here, attention is restricted to situations where $\mathcal{B}$ is spherical and where, for each time $t$, the deformation $\mathbf{y}(\cdot, t)$ is spherically symmetric and the solvent concentration $c(\cdot, t)$ is either uniform or piecewise uniform. These assumptions allow for an analytical treatment where time never appears explicitly and the constitutive response $\hat{\mathbf{M}}$ is never needed. In the subsequent analysis, we may therefore safely omit the variable $t$ and avoid any discussion regarding $\hat{\mathbf{M}}$. 


\section{Spherically symmetric problem}

We now suppose that $\mathcal{B}$ is a spherical solid specimen of radius $A$ in a spherically symmetric state. In other words, material points in a spherical ball of radius $R$, with $0 \leq R \leq A$, are mapped to a spherical ball with radius $r=f(R)$, with $f^{\prime}(R)>0$, where a prime denotes differentiation with respect to $R$, and have solvent concentration $c(R)$. Further, we assume that $\mathcal{B}$ remains intact after deformation, which requires

$$
f(0)=0 .
$$

The matrix $[\mathbf{F}]$ representing the deformation gradient $\mathbf{F}$ can then be written as

$$
[\mathbf{F}]=\operatorname{diag}\left\{\lambda_{R}, \lambda, \lambda\right\}
$$

where the principal stretches $\lambda_{R}$ and $\lambda$ are given in terms of $f$ by

$$
\lambda_{R}(R)=f^{\prime}(R), \quad \lambda(R)=\frac{f(R)}{R} .
$$

The relations (11) imply that the constraint (1) of incompressibility can be expressed as

$$
\frac{f^{2}(R) f^{\prime}(R)}{R^{2}}=1+v c(R) .
$$

Upon integrating (12) with respect to $R$ and using the condition (9), it follows that

$$
f^{3}(R)=3 \int_{0}^{R} x^{2}(1+v c(x)) d x .
$$

Further, since the principal stretches must be positive, (13) and $(11)_{2}$ imply that

$$
R \lambda(R)=\left(3 \int_{0}^{R} x^{2}(1+v c(x)) d x\right)^{1 / 3} .
$$

It is worth emphasizing the importance of expression (13). It dictates that a spherically symmetric state is fully determined by the knowledge of the solvent concentration field $c$. In view of this, let us suppose that $\mathcal{B}$ is in a state for which $c$ is known and the deformation is obtained via (13). Then $(2)_{1}$ and (8) determine the Lagrange multiplier field $q$ in a way that ensures mechanical equilibrium.

We now consider the Piola stress $\mathbf{S}$, bearing in mind the assumption that $c$ is known. On using (8), it follows that $\mathbf{S}$ can be represented by the matrix

$$
[\mathbf{S}]=\operatorname{diag}\left\{s_{R}, S, S\right\},
$$

with $s_{R}$ and $s$ determined by

$$
s_{R}=\alpha_{1} \lambda_{R}+2 \alpha_{2} \lambda^{2} \lambda_{R}^{2}-\frac{q}{\lambda_{R}}, \quad s=\alpha_{1} \lambda+\alpha_{2}\left(\lambda_{R}^{2}+\lambda^{2}\right) \lambda^{2}-\frac{q}{\lambda} .
$$

Take note that (15) informs us that, in a spherically symmetric state, the surface traction at the solid-environment interface is a normal traction. This implies that the traction boundary condition (4) $)_{1}$ requires the specification of $s_{R}$ at $R=A$.

We assume that $\mathcal{B}$ is acted upon by the ambient pressure $p_{a}$ and a normal Piola traction $\sigma$, which implies that the boundary conditions in (4) can be written as

$$
s_{R}(A)=\sigma-p_{a} \lambda^{2}(A), \quad \mu(A)=\mu_{a} .
$$

In view of $(3)_{3},(16)_{1}$, and (17), it follows that the values of $s_{R}$ and $\mu$ at $R=A$ involve $q(A)$ and consistent with the penultimate sentence of the paragraph including (1)-(3), thereby provide a coupling between mechanics and chemistry via boundary conditions. These boundary conditions can be combined to eliminate $q(A)$, yielding the expression

$$
\sigma=\alpha_{1} \lambda_{R}(A)+2 \alpha_{2} \lambda^{2}(A) \lambda_{R}^{2}(A)+\left.\frac{\lambda^{2}}{v} \frac{\partial \check{\psi}\left(\lambda_{R}(A), \lambda(A), C\right)}{\partial C}\right|_{C=c(A)}+\left(p_{a}-\frac{\mu_{a}}{v}\right) \lambda^{2}(A),
$$

for the prescribed traction $\sigma$, where $\check{\psi}$ is defined by

$$
\check{\psi}\left(\lambda_{R}, \lambda, c\right):=\hat{\psi}(\mathbf{F}, c) \text {. }
$$

Notice that (18) offers a relation between solvent concentration, prescribed traction, and environmental variables. This relation also involves the constitutive response $\hat{\psi}$ and the parameter $v$.

For the environment describe above, it easy to see that the net-potential energy (6) can be written as

$$
\mathcal{F}(c)=\mathcal{E}(c)+\mathcal{L}(c),
$$


with the canonical free-energy $\mathcal{E}$ given by

$$
\mathcal{E}(c)=4 \pi \int_{0}^{A}\left(\bar{\psi}(\lambda(R), c(R))-\mu_{a} c(R)\right) R^{2} d R,
$$

where $\bar{\psi}$ is defined as

$$
\bar{\psi}(\lambda, c):=\check{\psi}\left(\frac{1+v c}{\lambda^{2}}, \lambda, c\right),
$$

and the net potential energy $\mathcal{L}$ given by

$$
\mathcal{L}(c)=4 \pi A^{3}\left(\frac{p_{a}}{3}\left(\lambda^{3}(A)-1\right)-\sigma(\lambda(A)-1)\right) .
$$

Although, as previously noted, a spherically symmetric state is fully determined by the knowledge of the solvent concentration field $c$ and mechanical equilibrium in bulk is trivially satisfied by a suitable choice of the Lagrange multiplier field $q$, the relation (18)-which involves the value of solvent concentration at the solid-environment interface, the prescribed traction $\sigma$, and the environmental variables $p_{a}$ and $\mu_{a}-$ must be satisfied. As discussed next, this relation can be used to characterize certain types of states.

\subsection{States with piecewise uniform solvent concentration}

Consider a state with piecewise uniform solvent concentration, that is, a state for which $c$ has the form

$$
c(R)= \begin{cases}c_{i}, & 0 \leq R<R_{*}, \\ c_{0}, & R_{*}<R \leq A,\end{cases}
$$

with $c_{i}$ and $c_{o}$ constant and $0<R_{*}<A$. Observe that $R_{*}$ locates an interface that separates $\mathcal{B}$ into two regions, an inner one with solvent concentration $c=c_{i}$ and an outer one with solvent concentration $c=c_{0}$.

On inserting (24) into (13), it follows that $f$ has the form

$$
f^{3}(R)= \begin{cases}\left(1+v c_{i}\right) R^{3}, & 0 \leq R<R_{*}, \\ \left(1+v c_{i}\right) R_{*}^{3}+\left(1+v c_{o}\right)\left(R^{3}-R_{*}^{3}\right), & R_{*} \leq R \leq A .\end{cases}
$$

In view of (11), (12), and (14), (25) implies that $\lambda(A)$ and $\lambda_{R}(A)$ satisfies the relations

$$
A \lambda(A)=\left(\left(1+v c_{i}\right) R_{*}^{3}+\left(1+v c_{o}\right)\left(A^{3}-R_{*}^{3}\right)\right)^{1 / 3}, \quad \lambda_{R}(A) \lambda^{2}(A)=1+v c_{o} .
$$

The state defined by (24) is characterized by three quantities, namely $c_{i}, c_{o}$, and $R_{*}$. In this regard, we consider two cases:

Case 1: $c_{i}=c_{o}$, with $c_{o}$ to be determined.

Case 2: $c_{i}$ and $R_{*}$ known and $c_{o}$ to be determined.

In both of these cases, $c_{o}$ can be determined as follows. Bearing in mind (10) and (26), it is easy to see that (18) yields an equation which involves $c_{o}$. The value of $c_{o}$ is then obtained as a solution, if it exists, to this equation.

Observe that Case 1 corresponds to a state with uniform solvent concentration. Further, it follows from (13) that

$$
f(R)=\left(1+v c_{o}\right)^{1 / 3} R,
$$

which on using (11) implies that the stretches $\lambda_{R}$ and $\lambda$ are constant, given by

$$
\lambda_{R}(A)=\lambda(A)=\left(1+v c_{o}\right)^{1 / 3},
$$

and, thus, equal. Notice that, in this case, the deformation of $\mathcal{B}$ is homogeneous and corresponds to an uniform expansion. Therefore, the state under consideration is an equilibrium state, a conclusion which follows from the general observation that a state with homogeneous deformation is necessarily an equilibrium state. Therefore, $c_{o}$ is a critical point of net potential energy, which, when scaled by $4 \pi A^{3}$, can be written as

$$
F\left(c_{o}\right)=h\left(\lambda_{o}\right)-\sigma\left(\lambda_{o}-1\right),
$$

where

$$
h\left(\lambda_{o}\right)=\frac{1}{3}\left[\bar{\psi}\left(\lambda_{o}, \frac{\lambda_{o}^{3}-1}{v}\right)+\left(\lambda_{o}^{3}-1\right)\left(p_{a}-\frac{\mu_{a}}{v}\right)\right], \quad \lambda_{o}=\left(1+v c_{o}\right)^{1 / 3} .
$$

Thus, $c_{o}$ can be obtained by solving the equation $d F\left(c_{o}\right) / d c_{o}=0$. Further, if $d^{2} F\left(c_{o}\right) / d c_{o}^{2} \geq 0$, the $c_{o}$ defines a stable state. Otherwise, it defines an unstable state.

In closing this subsection, it is important to clarify one point. We have considered states distinguished by whether the solvent concentration is uniform or piecewise uniform. These states satisfy the boundary conditions given by (17). In particular, chemical equilibrium was assumed to prevail at the solid-environment interface. For a state with uniform solvent concentration (Case 1), it was shown that chemical equilibrium was also satisfied in bulk. Nothing was, or actually could 
be, said with respect to the chemical nature of a state with piecewise uniform composition (Case 2). This would require a more detailed analysis within the realm of swelling kinetics. So, instead of pursuing the complex task of discussing swelling kinetics, we assumed that a state with piecewise uniform composition to be attained during swelling-leaving circumstances under which this assumption is holds to be determined. Nevertheless, states with piecewise uniform composition have been the basis for theoretical studies of volumetric transitions in gels (see, for instance, Dolbow et al. (2004)). It also appears in the context of diffusion of liquids in polymers (see the review of Vesely (2008) and references cited therein).

\section{Cavitation}

Consider the solid sphere $\mathcal{B}$ in an intact state, that is, a state for which $\mathcal{B}$ remains cavity-free after deformation. We now investigate the possibility of bifurcation from such a state to a cavitated state, namely to a state consistent with the opening of a cavity in the center of the specimen. This being so, $f$ no longer obeys the condition (9). Towards this end, we assume that the cavitation process occurs so rapidly that there is insufficient time for redistribution of the solvent to occur, that mechanical equilibrium is attained instantaneously, and that the cavity is traction-free.

Granted these assumptions, the cavitated state arising from an intact state with solvent concentration $c$ has deformation obeying the relation

$$
f^{3}(R)=r_{c}^{3}+\int_{0}^{R} x^{2}(1+v c(x)) d x,
$$

which is obtained by integrating (12), where the cavity radius

$$
r_{c}:=f(0)>0
$$

is a constant to be determined. Further, by (11) and (31), the stretch $\lambda$ is now given by

$$
R \lambda(R)=\left(r_{c}^{3}+\int_{0}^{R} x^{2}(1+v c(x)) d x\right)^{1 / 3}
$$

The cavity radius $r_{c}$ is determined by using the mechanical equilibrium condition, the boundary condition (17) ${ }_{1}$, and the requirement that the cavity be traction-free-that is, by solving the purely mechanical part of the problem. Notice that the chemical part of the problem is trivial since the solvent concentration remains unaltered. To deal with mechanical equilibrium, it is convenient to introduce the total energy $U$ associated with cavitated states. Thanks to the traction-free cavity condition, this energy has the same form as the net potential energy in (20), except for the term involving $\mu_{a}$. Further, in view of relation (32) and since $c$ is assumed to be known, $U$ is as a function of $r_{c}$ only. Therefore, the cavity radius $r_{c}$ is obtained by solving the equation $d U\left(r_{c}\right) / d r_{c}=0$. On using (14), (21), and (23), this implies that, whenever $r_{c}>0$,

$$
\sigma=\lambda^{2}(A) \int_{0}^{A} \frac{1}{R \lambda^{2}} \frac{\partial \bar{\psi}(\lambda, c)}{\partial \lambda} d R+\lambda^{2}(A) p_{a},
$$

where $\lambda$ and $c$ are functions of $R$.

To evaluate the integral in (33), it is convenient to assume that the mapping $R \mapsto \lambda(R)$ is invertible, which allows a change of variable in the integral in (33) from $R$ to $\lambda$. In this case, (33) can be rewritten as

$$
\sigma=\lambda^{2}(A) \int_{\infty}^{\lambda(A)} \Psi(\lambda, c) d \lambda+\lambda^{2}(A) p_{a},
$$

where the integrand $\Psi$ is defined by

$$
\Psi(\lambda, c):=\frac{1}{1+v c-\lambda^{3}} \frac{\partial \bar{\psi}(\lambda, c)}{\partial \lambda},
$$

with the understanding that $c$ is now to be viewed as a function of $\lambda$. Notice that cavitation is possible provided that integral in (34) is finite, which in turn depends on the behavior of $\bar{\psi}$ for large values of $\lambda$. It is worth remarking that the integral on the right-hand side of (34) is obtained following ideas introduced by Ball (1982) and used in many subsequent works on cavitation. In particular, the steps necessary to arrive at (34) are essentially the same as those employed by Pence and Tsai (2006).

Following Ball (1982), we define the critical Piola traction $\sigma_{\mathrm{cr}}$ as the value of the applied Piola traction $\sigma$ at which an intact state $\left(r_{c}=0\right)$ bifurcates to a cavitated state $\left(r_{c}>0\right)$. This quantity is obtained by setting $r_{c}=0$ in (34), which yields

$$
\sigma_{\mathrm{cr}}=\zeta^{2} \int_{\infty}^{\zeta} \Psi(\lambda, c) d \lambda+\zeta^{2} p_{a}, \quad A \zeta=\int_{0}^{A} x^{2}(1+v c(x)) d x
$$

For later reference, we now specialize (34) to the case where the solvent concentration has the piecewise uniform form (24), yielding

$$
\sigma=\lambda^{2}(A) \int_{\infty}^{\lambda\left(R_{*}\right)} \Psi\left(\lambda, c_{i}\right) d \lambda+\lambda^{2}(A) \int_{\lambda\left(R_{*}\right)}^{\lambda(A)} \Psi\left(\lambda, c_{o}\right) d \lambda+\lambda^{2}(A) p_{a}
$$


where, on using (24) in (32),

$$
R_{*} \lambda\left(R_{*}\right)=\left(r_{c}^{3}+\left(1+v c_{i}\right) R_{*}^{3}\right)^{1 / 3}
$$

and

$$
A \lambda(A)=\left(r_{c}^{3}+\left(1+v c_{i}\right) R_{*}^{3}+\left(1+v c_{o}\right)\left(A^{3}-R_{*}^{3}\right)\right)^{1 / 3} .
$$

Notice that when $c_{i}=c_{0}$, relation (37) further simplifies to

$$
\sigma=\lambda^{2}(A) \int_{\infty}^{\lambda(A)} \Psi\left(\lambda, c_{0}\right) d \lambda+\lambda^{2}(A) p_{a},
$$

with

$$
A \lambda(A)=\left(r_{c}^{3}+\left(1+v c_{o}\right) A^{3}\right)^{1 / 3} .
$$

So far, nothing has been said about the nature of the intact state. We are interested in states attained during the uptake of a swelling agent by the solid sphere $\mathcal{B}$. This implies that relation (18) must play an important role in the cavitation analysis carried out here.

\section{Applications}

Suppose that $\mathcal{B}$ is a polymer network that can absorb solvent molecules identified with a fixed reference configuration free of solvent molecules (that is, with a dry polymer network).

We now present applications of a special version of the theory presented here. This specialization is based on the following assumptions:

- The solid sphere is immersed in a pure and incompressible liquid solvent at fixed pressure $p_{a}$. This implies that the chemical potential (of the swelling agent) in this environment can be expressed as

$$
\mu_{a}=\mu^{0}+v p_{a}
$$

- The free-energy density $\tilde{\psi}$ takes into account three contributions: the energy of the "unmixed" pure solvent, the elastic energy due to network deformation, and the energy of mixing. Invoking the Frenkel-Flory-Rehner hypothesis (see, for example, Flory, 1953), we assume that the latter two contributions are additive and separable. It then follows that

$$
\hat{\psi}(\mathbf{F}, c)=\mu^{0} c+\tilde{\psi}_{e}(\mathbf{B})+\tilde{\psi}_{m}(c),
$$

where the elastic and mixing contributions are given by

$$
\tilde{\psi}_{e}(\mathbf{B})=\frac{1}{2} c_{S} k_{B} T(\operatorname{tr} \mathbf{B}-3),
$$

with $\mathbf{B}=\mathbf{F F}^{\top}$, and

$$
\tilde{\psi}_{m}(c)=k_{B} T c(\ln (1-\phi)+\chi \phi), \quad \phi=\frac{1}{1+v c} .
$$

Here and throughout this paper, $\mu^{0}$ is a reference value of the chemical potential, $c_{S}$ is the cross-link density, $k_{B}$ is Boltzmann's constant, $T$ is the absolute temperature, $\phi$ is the polymer volume-fraction, and $\chi$ is the Flory-Huggins interaction parameter. The elastic contribution corresponds to Gaussian network theory (see, for example, Treloar, 1975), whereas the mixing contribution is given by the Flory-Huggins expression (see, for instance, Flory, 1953). The parameter $\chi$, which characterizes the interaction between polymer and solvent molecules, is positive for attraction and negative for repulsion.

Taking into account the aforementioned assumptions, it is easy to conclude that $\bar{\psi}$ in (22) reduces to

$$
\bar{\psi}(\lambda, c)=\mu^{0} c+\frac{c_{S} k_{B} T}{2}\left(\frac{1}{\lambda^{4} \phi^{2}}+2 \lambda^{2}-3\right)+k_{B} T c(\ln (1-\phi)+\chi \phi),
$$

with $\phi$ given by $(45)_{2}$. In addition, (18) reduces to

$$
\sigma=c_{S} k_{B} T \lambda_{R}(A)+\frac{k_{B} T}{v}\left(\ln (1-\phi(A))+\phi(A)+\chi \phi^{2}(A)\right) \lambda^{2}(A)
$$

The second term on the right-hand side of (47) is the mixing contribution to the osmotic pressure multiplied by $-\lambda^{2}$. Therefore, the applied Piola traction $\sigma$ is balanced by the difference between an elastic traction due to the network and an osmotic pressure due to the mixing. In this regard, notice that $c_{S} v$ measures the relative importance of these two contributions. As can be easily seen, the lower the value of $c_{S} v$, the more important is the osmotic contribution and, for a fixed value of $v$, the softer is the solid. 


\subsection{Uniform equilibrium states}

We now consider the problem of determining states for which $c=c_{o}$, where $c_{o}$ is a constant to be deduced.

Following the discussion of Section 3.1, the use of (27) in (47) gives the equation that must be solved for $c_{o}$. On using the polymer volume-fraction

$$
\phi=\frac{1}{1+v c_{o}},
$$

this equation is conveniently written as

$$
\sigma=\hat{\sigma}(\phi):=\frac{k_{B} T}{v}\left(c_{S} v \phi^{-1 / 3}+\left(\ln (1-\phi)+\phi+\chi \phi^{2}\right) \phi^{-2 / 3}\right) .
$$

Therefore, to determine the equilibrium values of the polymer volume-fraction $\phi$, we must solve the Eq. (49). A solution $\phi$ to (49) is stable whenever $\hat{\sigma}^{\prime}(\phi) \leq 0$ and unstable otherwise.

We begin by investigating the issue of existence of solutions to (49). We observe that $\hat{\sigma}$ is continuous on the interval $(0,1)$, that

$$
\lim _{\phi \rightarrow 0} \hat{\sigma}(\phi)=+\infty
$$

and that

$$
\lim _{\phi \rightarrow 1} \hat{\sigma}(\phi)=-\infty
$$

Therefore, $\hat{\sigma}$ maps the interval $(0,1)$ onto the real line. This implies that (49) admits a solution $\phi_{e}$ for any choice of $\sigma$. As discussed previously, such a solution is stable only if $\hat{\sigma}^{\prime}\left(\phi_{e}\right) \leq 0$. Multiple solutions are possible whenever $\hat{\sigma}^{\prime}>0$ holds over a subset of $(0,1)$. Hence, the behavior of $\hat{\sigma}$ is crucial for the analysis of both the stability and the uniqueness of solutions. In what follows, we investigate how the behavior of $\hat{\sigma}^{\prime}$ is affected by the parameters $c_{S}, v$, and $\chi$.

To investigate the influence of the parameters $c_{S}, v$, and $\chi$ on the behavior of $\hat{\sigma}^{\prime}$, we begin by introducing

$$
\hat{\chi}\left(\phi, c_{S} v\right):=\frac{3}{4(1-\phi)}+\frac{1}{2} \phi^{-2}(\ln (1-\phi)+\phi)+\frac{c_{S} v}{4} \phi^{-5 / 3} .
$$

A simple calculation using (49) shows that, for a given $c_{S} v, \hat{\sigma}^{\prime}(\phi)$ and

$$
\chi-\hat{\chi}\left(\phi, c_{S} v\right)
$$

have the same sign. Moreover, for each $c_{S} v, \hat{\chi}$ has the property that its minimum is attained at a value $\phi_{c}$ of $\phi$ in $(0,1)$ - that is, $\chi_{c}:=\hat{\chi}\left(\phi_{c}, c_{S} v\right) \leq \hat{\chi}\left(\phi, c_{S} v\right)$ for all $\phi$ in $(0,1)$. As a result, it follows that, if $\chi \leq \chi_{c}$, then $\hat{\sigma}^{\prime}(\phi) \leq 0$ for all $\phi$ in $(0,1)$, which implies that $\hat{\sigma}$ decreases monotonically. Otherwise, if $\chi>\chi_{c}$, then, by continuity, $\hat{\sigma}^{\prime}(\phi) \leq 0$ and $\hat{\sigma}^{\prime}(\phi)>0$ over two nonempty and complementary portions of $(0,1)$, which implies that $\hat{\sigma}$ is nonmonotonic. These conclusions are depicted in Fig. 1 , which shows the behavior of $\hat{\sigma}^{\prime}$ in the $(\phi, \chi)$-diagram for a fixed value of $c_{S} v$. It contains two complementary regions in which $\hat{\sigma}^{\prime}<0$ (the stable regime) and $\hat{\sigma}^{\prime}>0$ (the unstable regime) separated by the set of points for which $\hat{\sigma}^{\prime}=0$. In addition, it shows that the condition $\chi<\chi_{c}$ implies that $\hat{\sigma}^{\prime}<0$ and, consequently, that $\hat{\sigma}$ is monotonically decreasing and therefore that (49) admits a unique and stable solution for a given $\sigma$. On the other hand, for $\chi>\chi_{c}$, the monotonicity of $\hat{\sigma}$ is lost, which indicates the possibility of multiple solutions. This fact is better illustrated in Fig. 2, which depicts a typical $(\sigma, \lambda)$-diagram for $\chi>\chi_{c}$, in which case $\phi_{1}$ and $\phi_{2}$ satisfy the conditions $\hat{\sigma}^{\prime}(\phi)=0, \sigma_{1}=\hat{\sigma}\left(\phi_{1}\right)$, and $\sigma_{2}=\hat{\sigma}\left(\phi_{2}\right)$. Using Fig. 2, it follows that:

- If $\sigma<\sigma_{1}$ or $\sigma>\sigma_{2}$, (49) admits a unique and stable solution. Notice that $\sigma_{1}>0$, which implies that $\sigma \leq 0$, is sufficient for unique and stable solutions. In other words, the solution is unique for both free swelling $(\sigma=0)$ and compressive $(\sigma<0)$ values of the applied Piola traction.

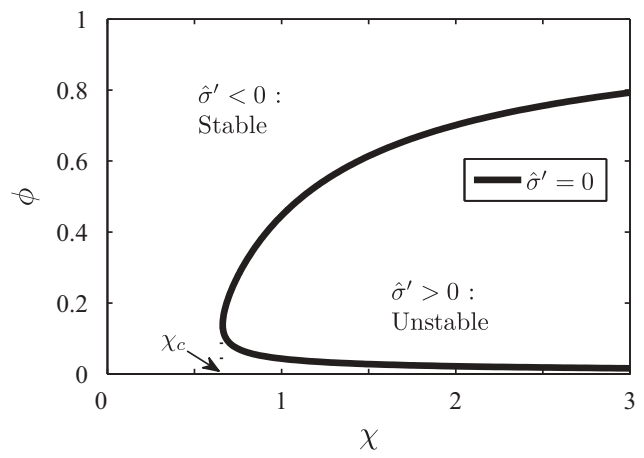

Fig. 1. $(\phi, \chi)$-diagram at constant $c_{S} v=0.01$, with the solid line separating unstable and stable regimes. 


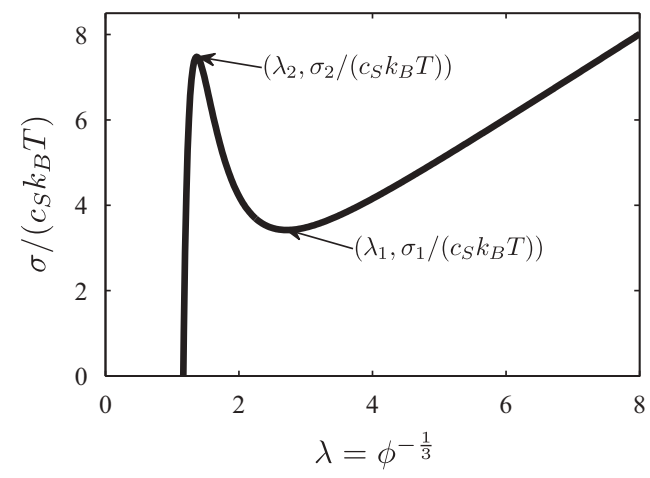

Fig. 2. $(\sigma, \lambda)$-diagram at constant $c_{S} v=0.01 . \phi_{1}$ and $\phi_{2}$ are such that $\sigma_{1}=\hat{\sigma}\left(\phi_{1}\right)$ and $\sigma_{2}=\hat{\sigma}\left(\phi_{2}\right)$.

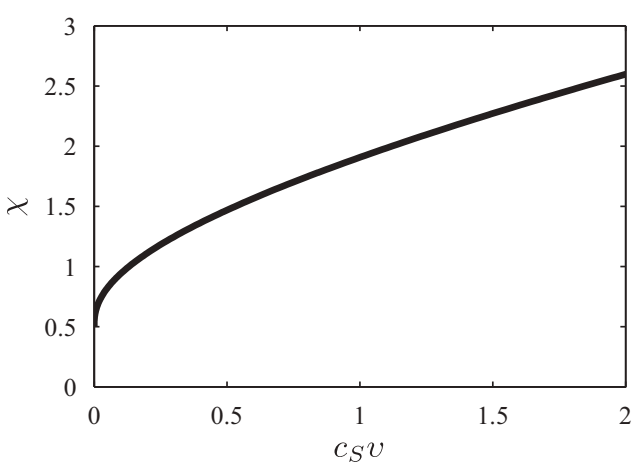

Fig. 3. $\left(\chi, c_{S} v\right)$-diagram in which the solid line represents the set of points for which $\chi=\chi_{c}$. Phase transformation is impossible for points below the solid line.

- If $\sigma=\sigma_{1}$ or $\sigma=\sigma_{2}$, (49) admits two solutions, both stable.

- If $\sigma_{1}<\sigma<\sigma_{2},(49)$ admits three solutions, two stable and one unstable.

Now we investigate how $\chi_{c}$ varies with $c_{S} v$. As can be seen in Fig. $3, \chi_{c}$ increases monotonically with $c_{S} v$ and is bounded below by the value $1 / 2$ corresponding to the unattainable limit where the polymer network vanishes. Considering $T$ and $v$ to be fixed, this implies that the softer the solid (that is, the lower the shear modulus $c_{S} k_{B} T$ ), the lower is the threshold $\chi_{c}$ demarking the boundary between unique and multiple solutions to (49).

For a given set of environmental conditions, the existence of two distinct values for the polymer volume-fraction $\phi$ associated with stable equilibrium states indicates the possibility of a volumetric phase-transformation between swollen and shrunken phases, which are defined by the lower and higher values of the equilibrium polymer volume-fraction, respectively.

\subsection{Cavitation of uniform equilibrium states}

We now consider the problem of cavitation of uniform equilibrium states. Toward this end, we begin by observing, after taking into account (35), (46), that (40) can be conveniently written as

$$
\sigma=c_{S} k_{B} T\left(\frac{1}{2 \phi \lambda^{2}(A)}+2 \lambda(A)\right)+p_{a} \lambda^{2}(A)
$$

where the polymer volume-fraction $\phi$ is as defined in (48) and the value $\lambda(A)$ of the stretch $\lambda$ at the boundary of the solid is given by (41). Therefore, for a given value of the applied Piola traction $\sigma$, the cavitation problem considered here consists in solving (49) and (51) for $\phi$ and $r_{c}$. We investigate the issue of existence and uniqueness for this problem with an emphasis on how this issue is influenced by the chemical parameters $c_{S}, v$, and $\chi$.

First, we examine the existence of solutions to (49) and (51) with $r_{c}=0$, which indicates that nucleation of a central cavity may occur. After setting $r_{c}=0$ in (41), it follows from these that nucleation is possible provided that $\phi$ satisfies the equation

$$
\ln (1-\phi)+\phi+\chi \phi^{2}=\gamma+\frac{3 c_{S} v}{2} \phi^{1 / 3},
$$


or, equivalently,

$$
\chi=\tilde{\chi}\left(\phi, c_{S} v, \gamma\right):=\frac{1}{\phi^{2}}\left(\gamma+\frac{3 c_{S} v}{2} \phi^{1 / 3}-\operatorname{In}(1-\phi)-\phi\right),
$$

where

$$
\gamma=\frac{v p_{a}}{k_{B} T}
$$

The critical value $\sigma_{\mathrm{cr}}$ of the applied Piola traction, which now is found by setting $r_{c}=0$ in (51), is given by

$$
\sigma_{\mathrm{cr}}=\frac{5}{2} c_{S} k_{B} T \phi^{-1 / 3}+p_{a} \phi^{-2 / 3} \text {. }
$$

It follows immediately that the following facts hold on the interval $(0,1)$ :

- The right-hand side of (52) is always positive, whereas the left-hand side of (52) is strictly negative whenever $\chi \leq 1 / 2$,

- $\tilde{\chi}\left(\cdot, c_{S} v, \gamma\right)$ is continuous and

$$
\lim _{\phi \rightarrow 0} \tilde{\chi}\left(\phi, c_{S} v, \gamma\right)=\lim _{\phi \rightarrow 1} \tilde{\chi}\left(\phi, c_{S} v, \gamma\right)=+\infty,
$$

which imply that $\tilde{\chi}\left(\cdot, c_{S} v, \gamma\right)$ has a minimum, say $\chi_{\mathrm{cr}}\left(c_{S} v, \gamma\right)$.

From these facts, we conclude that a condition necessary and sufficient for the existence of a solution of (52) is given by

$$
\chi \geq \chi_{\mathrm{cr}}\left(c_{S} v, \gamma\right)>1 / 2 .
$$

On using the definition of $\tilde{\chi}$ and $\chi_{\mathrm{cr}}$, it can be shown that $\chi_{\mathrm{cr}}\left(c_{S} v, \cdot\right)$ increases with $\gamma$, whereas $\chi_{\mathrm{cr}}(\cdot, \gamma)$ decreases with $c_{S} v$. These facts are illustrated in Fig. 4.

We now turn to the issue of uniqueness of solutions to (52). As Fig. 5 indicates, for a given $\chi>\chi_{\mathrm{cr}}\left(c_{S} v, \gamma\right)$ equation $(52)$ admits two solutions, where-provided $\chi$ is sufficiently large-the largest (smallest) one corresponds to a stable (an unstable) solution for the intact sphere. Therefore, the smallest solution to (52), which corresponds to an unstable

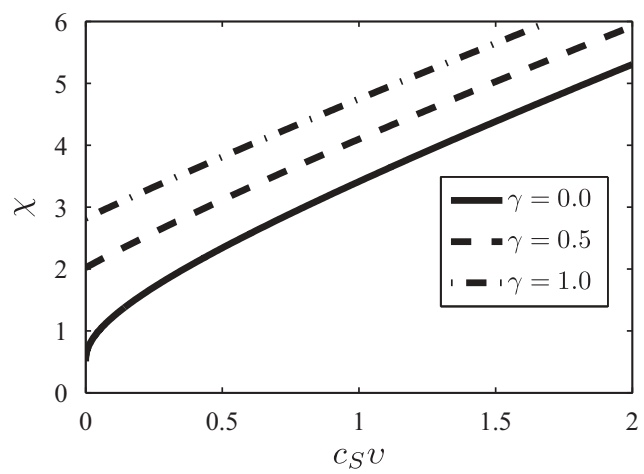

Fig. 4. $\left(\chi, c_{S} v\right)$-diagram in which lines represent the set of points for which $\chi=\chi_{\mathrm{cr}}\left(c_{S} v, \gamma\right)$. For a given value of $\gamma$, cavitation is impossible for points below the corresponding line.

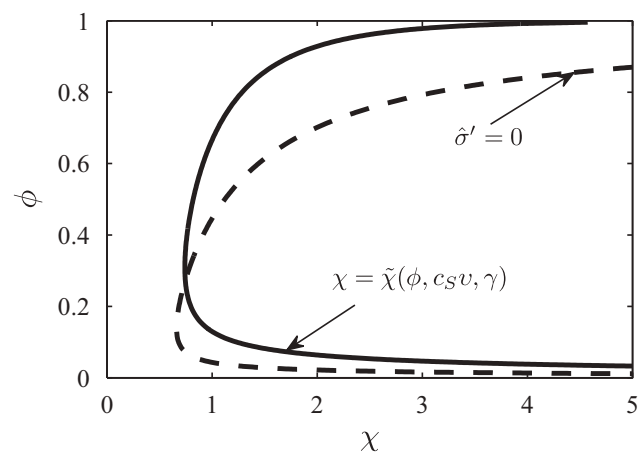

Fig. 5. $(\phi, \chi)$-diagram for a fixed value of $c_{S} v=0.01$ and $\gamma=0$. The solid line represents the solution of the nucleation condition. The dashed line separates stable and unstable regimes for the intact sphere. 
equilibrium state, is ruled out. Notice also that, for $\chi_{c}<\chi<\chi_{\mathrm{cr}}\left(c_{S} v, \gamma\right)$, multiple uniform equilibrium solutions are possible, but that the nucleation of a cavity is impossible.

Now we investigate the cavitation problem for $r_{c}>0$, taking advantage of the $(\sigma, \phi)$-diagram in Fig. 6 . This diagram shows two set of curves corresponding to two set of equations: (49) for two values of the interaction parameter $\chi$; (51) for different values of the cavity radius $r_{c}$. The second of these equations shows that $\sigma$ is an increasing function of $r_{c}$. Solutions for the cavitation problem correspond to intersections of these two sets of curves. Notice that the maximum value of $r_{c}$ is attained at $\sigma=\sigma_{2}$, with $\sigma_{2}$ defined in the previous subsection. This is also illustrated in Fig. 7 for $c_{S} v=0.01, \chi=1$, and $\gamma=0$. The existence of two "branches" of the curve is explained by the fact that for a given $r_{c}$ it is possible to have two associated values of $\phi$, the smallest one corresponding to an unstable intact state. Notice that the "lowest branch" corresponds to cavitation of unstable states.

The main results can be summarized as follows:

- The condition $\chi \geq \chi_{\mathrm{cr}}\left(c_{S} v, \gamma\right)>1 / 2$ is necessary for cavitation.

- If the above condition is verified, cavitation is possible only if $\sigma_{\mathrm{cr}}<\sigma<\sigma_{2}$.

\subsection{Cavitation of dry-swollen states}

We now consider the cavitation of a state with piecewise uniform solvent concentration for which $c_{i}=0, R_{*}$ is given, and $c_{o}$ is to be determined. In this case, the inner core of $\mathcal{B}$ is dry.

We begin by writing appropriate versions of Eqs. (37) and (47). On using (26) and invoking the definition (48) of $\phi$, it follows that (47) can be expressed as

$$
\sigma=\frac{k_{B} T}{v} l^{2}\left(\frac{c_{S} v}{\phi l^{4}}+\ln (1-\phi)+\phi+\chi \phi^{2}\right)
$$

with

$$
l=\phi^{-1 / 3}\left((\phi-1) \bar{R}_{*}^{3}+1\right)^{1 / 3}, \quad \bar{R}_{*}=\frac{R_{*}}{A} .
$$

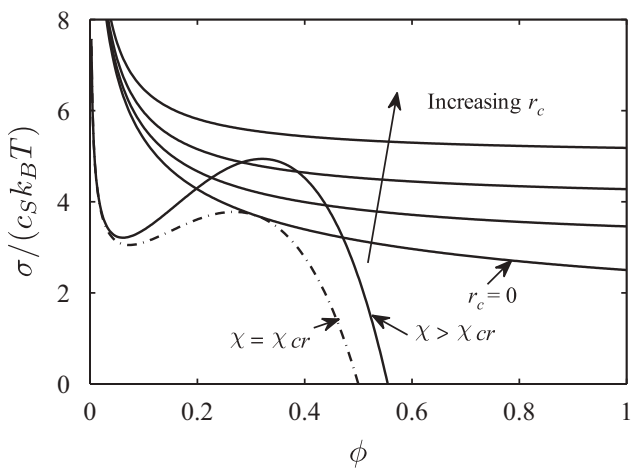

Fig. 6. $(\sigma, \phi)$-diagram for a fixed value of $c_{S} v$.

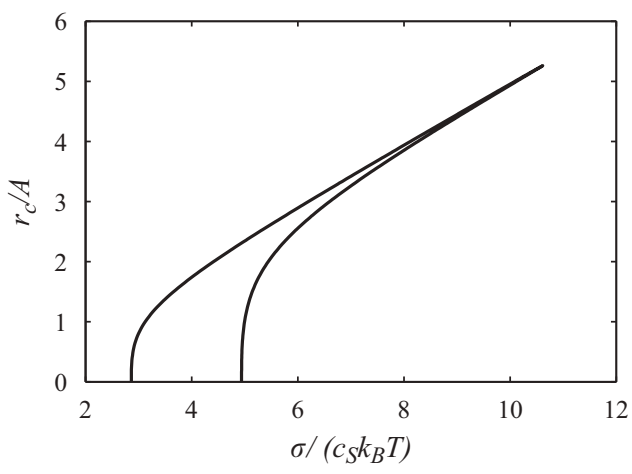

Fig. 7. $\left(\sigma, r_{c}\right)$-diagram for $c_{S} v=0.01, \chi=1$, and $\gamma=0$. 
Bearing in mind (46) it follows that (37) simplifies to

$$
\sigma=c_{S} k_{B} T \lambda^{2}(A)\left(\frac{1}{2 \lambda^{4}\left(R_{*}\right)}+\frac{2}{\lambda\left(R_{*}\right)}+\left[\frac{1}{2 \phi \lambda^{4}}+\frac{2}{\lambda}\right]_{\lambda\left(R_{*}\right)}^{\lambda(A)}+\frac{p_{a}}{c_{S} k_{B} T}\right),
$$

where

$$
\lambda\left(R_{*}\right)=\left(\left(\frac{\bar{r}_{c}}{\bar{R}_{*}}\right)^{3}+1\right)^{1 / 3}, \lambda(A)=\phi^{-1 / 3}\left(\phi\left(\bar{r}_{c}^{3}+\bar{R}_{*}^{3}\right)+1-\bar{R}_{*}^{3}\right)^{1 / 3}, \quad \bar{r}_{c}=\frac{r_{c}}{A} .
$$

Thus, the cavitation problem consists of solving (57) and (59) for $\phi$ and $r_{c}$. We investigate the critical conditions under which the nucleation of a cavity may occur.

The critical value $\sigma_{\mathrm{cr}}$ of the applied Piola traction, which is now found by setting $r_{c}=0$ in (59), is given by

$$
\sigma_{\mathrm{cr}}=c_{S} k_{B} T\left(\frac{\phi-1}{2 \phi} l^{2}+\frac{1}{2 \phi l^{2}}+2 l\right)+p_{a} l^{2}
$$

where $\phi$ is a solution to the equation

$$
\frac{c_{S} v}{\phi l^{4}}+\ln (1-\phi)+\phi+\chi \phi^{2}=\gamma+c_{S} v\left(\frac{\phi-1}{2 \phi}+\frac{1}{2 \phi l^{4}}+\frac{2}{l}\right)
$$

This equation results from the requirement that both (57) and (59) hold for $r_{c}=0$. In view of (58), (61), and (62), it easy to see that $\sigma_{\mathrm{cr}}$ is a function of $c_{S}, v, \chi, T, p_{a}$, and $R_{*}$. In what follows, we discuss how $\sigma_{\mathrm{cr}}$ is affected by $\chi$ and $R_{*}$. For illustrative purposes, this discussion assumes that $c_{S} v$ and $p_{o}$ are given by $c_{S} v=0.01$ and $p_{a}=0$.

Fig. 8 shows how $\sigma_{\mathrm{cr}}$ varies with $\chi$ for two different values of $R_{*}$. The solid line represents the set of points for which $\sigma=\sigma_{\mathrm{cr}}$ in the case $R_{*} / A=0.9$. This lines divides the $(\sigma, \chi)$-diagram in two regions, a lower region where cavitation is impossible and an upper region where cavitation may occur. Notice that the smaller the interaction parameter is, the lower is the value of the critical Piola traction $\sigma_{\mathrm{cr}}$. Further, as $\chi$ increases, the critical Piola traction $\sigma_{\mathrm{cr}}$ approaches the value $\left(5 c_{S} k_{B} T / 2\right)$ corresponding to the dry limit. Notice also that cavitation may occur even if the Piola traction $\sigma$ is zero or even negative. Whether this is possible depends on the value of the interaction parameter $\chi$. Fig. 9 shows the variation of $\sigma_{\mathrm{cr}}$ with the interface location for different values of $\chi$. Notice that critical Piola traction $\sigma_{\mathrm{cr}}$ diminishes with the advance of the

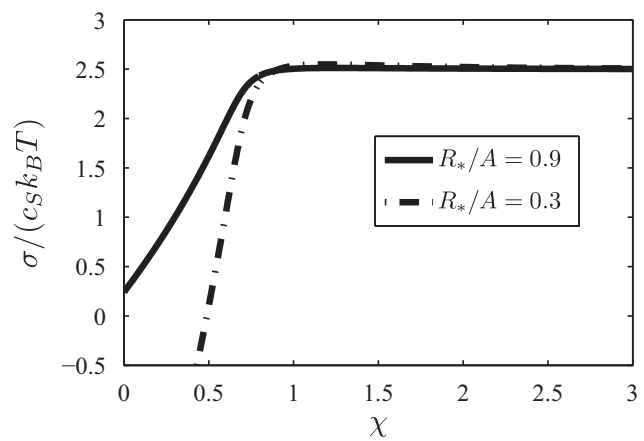

Fig. 8. $(\sigma, \chi)$-diagram for $c_{S} v=0.01, p_{a}=0$, and two different values of the normalized interface location $R_{*} / A$. For each value of $R_{*} / A$, the corresponding line represents the set of points for which $\sigma=\sigma_{\mathrm{cr}}$. Cavitation is impossible for $\sigma<\sigma_{\mathrm{cr}}$.

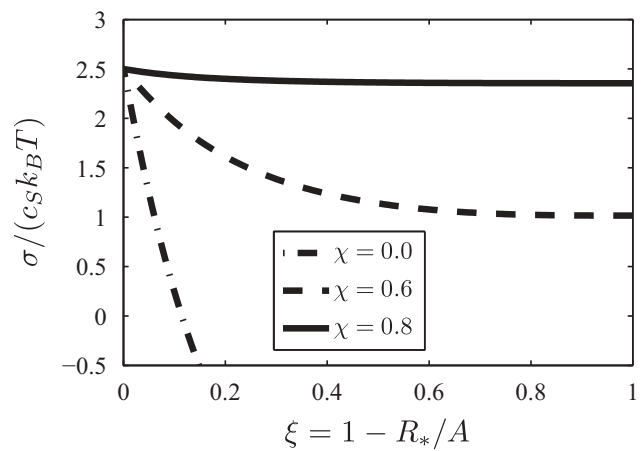

Fig. 9. $(\sigma, \xi)$-diagram for $c_{S} v=0.01, p_{a}=0$, and different values for the interaction parameter $\chi$. For each $\chi$, the corresponding line represents the set of points for which $\sigma=\sigma_{\mathrm{cr}}$. Cavitation is impossible for $\sigma<\sigma_{\mathrm{cr}}$. 
interface. Notice also that cavitation may occur even for non-positive values of $\sigma$. It is important to observe that Pence and Tsai (2006) presented a very detailed and general analysis concerning swelling-induced cavitation, that is, the possibility of cavitation with $\sigma=0$, for states with piecewise constant swelling.

\section{Conclusions}

In this work, we developed a treatment for investigating the role played by the mutual interaction between mechanics and the absorption of a swelling agent on the equilibrium and cavitation of swollen intact spheres. This treatment was then applied, within the context of the classical theory of the swelling of a rubber by a liquid, to describe two types of intact states of a spherical specimen immersed in a pure and incompressible liquid solvent. In one of these, the specimen inhabits a uniform equilibrium state. In the other, the specimen is divided in two regions, the inner one dry and the outer one uniformly swollen, separated by a sharp boundary. The effects of the constitutive parameters entering the theory-namely the number $c_{S}$ of polymer chains per unit reference volume, the volume $v$ occupied by a swelling-agent molecule, and the Flory-Huggins interaction parameter $\chi$-on the characteristics of the two types of intact states were also addressed. For uniform equilibrium states, it was shown that multiphase equilibria and cavitation are impossible unless the parameter $\chi$ exceeds a certain critical value that depends on both $c_{S} v$ and, granted that cavitation occurs, $\gamma=v p_{a} / k_{B} T$, where $p_{a}$ is the solvent pressure in the environment, $k_{B}$ is Boltzmann's constant, and $T$ is the absolute temperature. Further, the critical load for cavitation increases with $\chi$ and is bounded below by the critical load obtained when environmental effects are not considered. As for states with a dry core surrounded by a swollen annular zone, it was shown that cavitation may occur even for non-positive values of the applied Piola traction $\sigma$. Whether this is possible depends on the values of the interaction parameter $\chi$ and the interface location $R_{*}$.

The cavitation analysis carried out here proceeded along the lines established by Pence and Tsai (2006). Whereas Pence and Tsai (2006) took an a priori specified swelling field, which otherwise was general in the sense that no specific swelling mechanism needed to be invoked, here we considered the swelling field as being determined as a result of the simultaneous interaction between mechanics and absorption. In this regard, the present work and that of Pence and Tsai (2006) can be viewed as complementary.

\section{Acknowledgments}

The first two authors wish to thank CNPq (201080/2008-7) and CAPES (0720/08-9), respectively, for grating scholarships to support their postdoctoral training. The last author thanks the Chemical and Metallurgical Engineering section of the Natural Sciences and Engineering Research Council of Canada for its support under a Discovery Grant and the Mechanical Engineering Department at the Universidade Federal do Rio de Janeiro for its generous hospitality.

\section{References}

Ball, J.M., 1982. Discontinuous equilibrium solutions and cavitation in nonlinear elasticity. Philosophical Transactions of the Royal Society A. Mathematical, Physical, and Engineering Sciences 306, 557-611.

Baek, S., Pence, T.J., 2011. Inhomogeneous deformations of elastomer gels in equilibrium under saturated and unsaturated conditions. Journal of the Mechanics and Physics of Solids 59, 561-582.

Barriere, B., Leibler, L., 2003. Kinetics of solvent absorption and permeation through a higly swellable elastomeric network. Journal of Polymer Science B: Polymer Physics 41, 166-182.

Chester, S.A., Anand, L., 2010. A coupled theory of fluid permeation and large deformations for elastomeric materials. Journal of the Mechanics and Physics of Solids 58, 1879-1906.

Crank, J., 1990. The Mathematics of Diffusion, second ed. Oxford University Press.

Doi, M., 2009. Gel dynamics. Journal of the Physical Society of Japan 78 052001-1-052001-19.

Dolbow, J., Fried, E., Huidi, J., 2004. Chemically induced swelling of hydrogels. Journal of the Mechanics and Physics of Solids 52, 51-84.

Duda, F.P., Souza, A.C., Fried, E., 2010. Theory for species migration in a finitely strained solid with application to polymer network swelling. Journal of the Mechanics and Physics of Solids 58, 515-529.

Dusek, K., Patterson, D., 1968. Transition in swollen polymer networks induced by intramolecular condensation. Journal of Polymer Science A 6 , 1200-1216.

Erman, B., Flory, P.J., 1986. Critical phenomena and transitions in swollen polymer networks and in linear macromolecules. Macromolecules 19, 2342-2353.

Flory, P.J., 1953. Principles in Polymer Chemistry. Cornell University Press.

Fond, C., 2001. Cavitation criterion for rubber materials: a review of void-growth models. Journal of Polymer Science B: Polymer Physics 39, $2081-2096$.

Gent, A.N., 1970. Hypothetical mechanism of crazing in glassy plastics. Journal of Materials Science 5, 925-932.

Gent, A.N., Lindley, P.B., 1959. Internal rupture of bonded rubber cylinders in tension. Proceedings of the Royal Society A, Mathematical, Physical, and Engineering Sciences 249, 195-205.

Hirotsu, S., 1994. Static and time-dependent properties of polymer gels around the volume phase transition. Phase Transitions 47, 183-240.

Hong, W., Zhao, X., Zhou, J., 2008. Suo. Z., 2008. A theory of coupled diffusion and large deformation in polymeric gels. Journal of the Mechanics and Physics of Solids 56, 1779-1793.

Horgan, C.O., Polignore, D.A., 1995. Cavitation in nonlinearly elastic solids: a review. Applied Mechanics Reviews 48, 471-485.

Li, Y., Tanaka, T., 1992. Phase transitions of gels. Annual Review of Materials Science 22, 243-277.

Onuki, A., 1993. Theory of phase transtion in polymer gels. Advances in Polymer Science 109, 63-121.

Pence, T.J., Tsai, H., 2006. Swelling-induced cavitation of elastic spheres. Mathematics and Mechanics of Solids 11, 527-551.

Rajagopal, K.R., 2003. Diffusion through polymeric solids undergoing large deformations. Materials Science and Technology 19, 1175-1180.

Saylor, D.M., Richardson, D.C., Dair, B.J., Pollack, S.K., 2010. Osmotic cavitation of elastomeric intraocular lenses. Acta Biomaterialia 6, $1090-1098$. 
Scheirs, J., 2000. Compositional and Failure Analysis of Polymers. John Wiley \& Sons.

Shibayama, M., Tanaka, T., 1993. Volume phase transition and related phenomena of polymer gels. Advances in Polymer Science $109,1-62$. Tanaka, T., 1978. Collapse of gels and the critical endpoint. Physical Review Letters 40, 820-823.

Treloar, L.R.G., 1975. The Physics of Rubber Elasticity. Oxford University Press.

Vesely, D., 2008. Diffusion of liquids in polymers. International Materials Reviews 53, 299-315.

Werner, L., 2010. Glistenings and surface light scattering in intraocular lenses. Journal of Cataract \& Refractive Surgery 36, $1398-1420$. 